\title{
Factors of microinflammation in non- diabetic chronic kidney disease: a pilot study
}

\author{
Valerie Olivier ${ }^{1 *}$, Catherine Dunyach-Remy ${ }^{2}$, Pierre Corbeau ${ }^{3}$, Jean-Paul Cristol ${ }^{4,5}$, Thibault Sutra ${ }^{4,5}$,
} Stephane Burtey ${ }^{6}$, Jean-Philippe Lavigne ${ }^{2}$ and Olivier Moranne ${ }^{1,7}$

\begin{abstract}
Background: The relationships between digestive bacterial translocation, uremic toxins, oxidative stress and microinflammation in a population of chronic kidney disease (CKD) patients without metabolic nor inflammatory disease are unknown.

Methods: Bacterial translocation, uremic toxins, oxidative stress, and inflammation were assessed by measuring plasma levels of $16 \mathrm{~S}$ ribosomal DNA (16S rDNA), p-cresyl sulfate (PCS), indoxyl sulfate (IS), indole acetic acid (IAA), F2-isoprostanes, hsCRP and receptor I of TNFa (RITNFa) in patients without metabolic nor inflammatory disease. 44 patients with CKD from stage IIIB to $V$ and 14 controls with normal kidney function were included from the nephrology outpatients. 11 patients under hemodialysis (HD) were also included. Correlations between each factor and microinflammation markers were studied.
\end{abstract}

Results: 165 rDNA levels were not increased in CKD patients compared to controls but were decreased in HD compared to non-HD stage $V$ patients (4.7 (3.9-5.3) vs 8.6 (5.9-9.7) copies/ $\mu$ l, $p=0.002)$. IS, PCS and IAA levels increased in HD compared to controls (106.3 (73.3-130.4) vs $3.17(2.4-5.1) \mu \mathrm{mol} / \mathrm{l}, p<0.0001$ for IS; 174.2 (125-227.5) vs 23.7 (13.9-52.6) $\mu \mathrm{mol} / \mathrm{I}, p=0.006$ for PCS; and 3.7 (2.6-4.6) vs $1.3(1.0-1.9) \mu \mathrm{mol} / \mathrm{I}, p=0.0002$ for IAA). Urea increased in non-HD stage $V$ patients compared to controls (27.6 (22.7-30.9) vs $5.4(4.8-6.4) \mathrm{mmol} / \mathrm{l}, p<0.0001)$ and was similar in HD and in non-HD stage $V(19.3(14.0-24.0)$ vs $27.6(22.7-30.9) \mathrm{mmol} / \mathrm{l}, p=0.7)$. RITNFa levels increased in HD patients compared to controls (12.6 (9.6-13.3) vs $1.1(1.0-1.4) \mathrm{ng} / \mathrm{ml}, \mathrm{p}<0.0001)$; hsCRP levels increased in non-HD stage $V$ patients compared to controls $(2.9(1.4-8.5)$ vs $0.8(0.5-1.7) \mathrm{mg} / \mathrm{l}, p=0.01)$ and remained stable in HD patients (2.9 (1.4-8.5) vs 5.1 (0.9-11.5) $\mathrm{mg} / \mathrm{l}, p=1)$. F2-isoprostanes did not differ in CKD patients compared to controls. Among uremic toxins, IS and urea were correlated to RITNFa $(r=0.8, p<0.0001$ for both). PCS, IS and urea were higher in patients with hsCRP $\geqq 5 \mathrm{mg} / \mathrm{l}$ ( $p=0.01,0.04$ and 0.001 respectively). $16 \mathrm{~S}$ rDNA, F2-isoprostanes were not correlated to microinflammation markers in our study.

Conclusions: In CKD patients without any associated metabolic nor inflammatory disease, only PCS, IS, and urea were correlated with microinflammation. Bacterial translocation was decreased in patients under HD and was not correlated to microinflammation.

Keywords: CKD, Microinflammation, Oxidative stress, Uremic toxins, Bacterial translocation

\footnotetext{
* Correspondence: valerie.olivier@unige.ch

'Department of Nephrology - Dialysis - Apheresis, Caremeau Hospital,

University Montpellier-Nimes, CHU Nîmes, Nimes, France

Full list of author information is available at the end of the article
}

(c) The Author(s). 2020 Open Access This article is licensed under a Creative Commons Attribution 4.0 International License, which permits use, sharing, adaptation, distribution and reproduction in any medium or format, as long as you give appropriate credit to the original author(s) and the source, provide a link to the Creative Commons licence, and indicate if changes were made. The images or other third party material in this article are included in the article's Creative Commons licence, unless indicated otherwise in a credit line to the material. If material is not included in the article's Creative Commons licence and your intended use is not permitted by statutory regulation or exceeds the permitted use, you will need to obtain permission directly from the copyright holder. To view a copy of this licence, visit http://creativecommons.org/licenses/by/4.0/ The Creative Commons Public Domain Dedication waiver (http://creativecommons.org/publicdomain/zero/1.0/) applies to the data made available in this article, unless otherwise stated in a credit line to the data. 


\section{Background}

Decreased glomerular filtration rate has been described as an independent factor associated with a high risk of cardiovascular events and mortality even at mild to moderate stages of chronic kidney disease (CKD) [1]. In addition to the high prevalence of traditional cardiovascular risk factors as diabetes mellitus and hypertension in CKD patients, microinflammation could contribute to accelerated atherosclerosis, as in general population [2].

Microinflammation in CKD results from many determinants apart from decreased clearance of proinflammatory cytokines: frequent infections [3], chronic heart failure [4], membrane bioincompatibility in hemodialysis (HD) [5], lack of vitamin D [6], metabolic acidosis [7], oxidative stress, uremic toxins, insulin resistance [8] and associated dysimmune comorbidities. More recently, the gut has been described as a contributor to microinflammation in CKD [9]. The kidney-gut concept illustrates the complex relationships between gut, uremic toxins and microinflammation in CKD: Gut microbiota undergoes modifications in CKD due to increased gut intraluminal urea, leading to selection of urease and uricase expressing bacteria and bacteria possessing indole and p-cresol-forming enzymes [10]. These bacteria will produce tryptophan or phenylalanine-derived uremic toxins such as indoxyl sulfate (IS), indole acetic acid (IAA) and p-cresol sulfate further sulfated into p-cresyl sulfate (PCS), which are known to have proinflammatory effects [11]. Increased intraluminal urea leads also to altered intestinal permeability and therefore to bacterial translocation that will result in activation of immune system and microinflammation [9]. Oxidative stress in CKD is mainly explained by an increase of reactive oxygen species production by activation and upregulation of pathways like nicotinamide adenin dinucleotide phosphatase (NADPH) oxidase [12] and Nox (NADPH oxidase) 4, mitochondria respiratory chain, xanthine oxidase, lipoxygenases, uncoupled nitric oxide synthases, the dysfunction of anti-oxidant systems due to the loss of nephrons [13]. CKD is associated with increased levels of oxidative stress markers such as oxidized nucleic acid, protein and lipid at different stages $[14,15]$. Metabolic conditions such as insulin resistance and obesity, that are frequently present in CKD patients can even more enhance oxidative stress [12] and bacterial translocation [16, 17] and thus participate in microinflammation.

The relationships between bacterial translocation, uremic toxins, oxidative stress and microinflammation in a population of CKD patients are still unknown and need to be better described. The objective of the study is to understand the relationships between bacterial translocation, oxidative stress, uremic toxins and microinflammation in a selected population of consecutive patients from stage IIIB to end-stage kidney disease, without any associated metabolic nor inflammatory disease. The hypothesis is that, in CKD and in HD, enhanced bacterial translocation, oxidative stress and increased uremic toxins stimulate microinflammation.

\section{Methods \\ Population}

From December 2016 to June 2017, we consecutively enrolled all adult patients in the Department of Nephrology-Dialysis of the Nîmes University Hospital (France) for CKD from stage IIIB to V. HD patients were recruited in the same period in our department. A control population with normal kidney function were included from the nephrology outpatients followed up for an inactive nephrolithiasis disease.

The exclusion criteria were the presence of factors that may influence the inflammatory status or the bacterial translocation such as: diabetes mellitus, a body mass index (BMI) more than $30 \mathrm{~kg} / \mathrm{m}^{2}$, a liver failure, a left ventricular ejection fraction below $40 \%$, a history of inflammatory bowel disease, bariatric surgery or cancer, a recent history of chronic inflammatory disease, a current infection or a recent antibiotic treatment, a current immunosuppressive or immunomodulatory treatment, and a vascular catheter.

\section{Sociodemographic and clinical data}

Epidemiological and clinical data were gathered for all patients including age, sex, body mass index, blood pressure, history of cardiovascular events, inflammatory disease, treatment, causes of CKD, active consumption of tobacco and the Charlson comorbidity index. For HD patients the following clinical data were collected: dialysis vintage, ultrafiltration volume, intradialytic hypotension defined as a fall of $40 \mathrm{mmHg}$ of systolic blood pressure (SBP) or a SBP under $100 \mathrm{mmHg}$ or the presence of symptoms suggesting hypotension during the current HD session, residual renal function, dialysis procedure (hemodialysis or hemodiafiltration).

\section{Biological data}

The following biological data were gathered: creatinine and estimated glomerular filtration rate using the CKD EPI formula, urea, urinary albumin creatinine ratio, blood hemoglobin, natural vitamin $\mathrm{D}$, and albuminemia.

\section{Blood samples}

Remnant plasma and serum were obtained after routine exams from blood samples collected at the regular follow up visit for non-HD patients, and at the regular monthly blood analysis for HD patients. For HD patients, pre-HD blood samples were collected at the midweek dialysis session. Blood was collected after a fasting period of at least $4 \mathrm{~h}$, and patients were asked not to 
brush their teeth before blood collection to avoid physiological bacterial translocation. Plasma and serum were collected in EDTA-anticoagulant-treated tubes and dry tubes respectively after centrifugation and then stored at $-80^{\circ} \mathrm{C}$.

\section{DNA extraction and 16S rDNA real-time PCR}

DNA was extracted from $200 \mu \mathrm{l}$ of plasma using the $E Z 1^{\circ}$ DNA Tissue kit (Qiagen, Courtaboeuf, France) according to the manufacturer's recommendations. DNA was eluted in a $100 \mu \mathrm{l}$ final volume. A negative control was extracted from molecular biology grade pure water in the same conditions. Bacterial $16 \mathrm{~S}$ rDNA levels were measured by qPCR as described previously [18]. The assays were performed using a LightCycler 480 II (Roche). Absolute quantification analysis was performed with the Lightcycler 480 software (Roche), version 1.5, according to the manufacturer's recommendations.

\section{HsCRP, RITNFa}

The concentration of plasma hsCRP was determined by immuno-turbidimetry. Receptor I of TNF $\alpha$ (RITNF $\alpha$ ) plasmatic rates were measured as an inflammation marker with Quantikine ELISA Human TNF RI/ TNFRSF1A ELISA kit (R\&D SYTEMS, Minneapolis, United States). The dilution factor was 1:20 according to the manufacturer's recommendations.

\section{Uremic toxins}

Plasmatic PCS, IAA and IS were measured by high performance liquid chromatography as described previously [19].

\section{Oxidative stress}

Plasmatic F2-Isoprostanes were measured by chromatography-mass spectrometry as marker of oxidative stress [20].

\section{Statistical analysis}

Quantitative data are presented as median (interquartile range). Categorical data are given in frequency and percentage. Markers of bacterial translocation, oxidative stress, inflammation, uremic toxins (IS, PCS, IAA) and urea are expressed according to CKD stage as follows: CKD stage IIIB, CKD stage IV, CKD stage V and CKD stage $\mathrm{V}$ under HD. Due to the low number of patients in each group and the non-Gaussian distribution of the variables, non-parametric tests were used. Quantitative values according to CKD were compared as follows: bivariate group comparisons were performed with a Mann-Whitney test or a global test of Kruskal-Wallis with a multipair wise comparison from Dunn when more than 2 groups were compared. For categorical data a Chi squared test was used to compare groups. The correlations between bacterial translocation, urea, uremic toxins, oxidative stress and RITNF $\alpha$ as continuous variables were analyzed with a Spearman test. Considering hsCRP as a categorical data, we compared the bacterial translocation, urea, uremic toxins and oxidative stress levels in subjects with hsCRP $\geq 5 \mathrm{mg} / \mathrm{l}$ with those in subjects with hsCRP $<5 \mathrm{mg} / \mathrm{l}$. Statistical difference was set up at a $p$-value $<0.05$ for bilateral test. Statistical analysis was performed using GraphPad Prism version 7.

\section{Results}

\section{Demographic and clinical characteristics}

Sixty-nine patients were included in this study: 14 patients in the CKD stage IIIB group, 15 patients in the stage IV group and non-HD stage V group, 11 patients in the HD group and 14 patients in the control group (Fig. 1). The clinical characteristics and standard biological parameters are detailed in Table 1 . No statistical difference in term of clinical characteristics could be observed among the different groups except for BMI ( $p=$ $0.02)$, SBP $(p=0.04)$, and Charlson comorbidity index $(p<0.0001)$. BMI and systolic blood pressure were low in HD group and Charlson comorbidity index was particularly high in stage $\mathrm{V}$ group, HD and non-HD.

\section{Bacterial translocation, uremic toxins and oxidative stress according to CKD stage}

For each CKD group, bacterial translocation was assessed by plasmatic $16 \mathrm{~S}$ rDNA quantification, uremic toxins: IS, PCS, IAA and urea were measured, and oxidative stress was assessed by F2-isoprostanes plasma quantification (Fig. 2). 16S rDNA levels were not increased in non-HD CKD patients compared to controls (8.6 (5.9-9.7) in nonHD stage V vs $5.9(5.2-7.0)$ copies $/ \mu$ in controls, $p=0.4)$. Surprisingly, plasmatic $16 \mathrm{~S}$ rDNA decreased in HD patients compared to non-HD stage $\mathrm{V}$ patients (4.7 (3.9-5.3) in HD patients vs $8.6(5.9-9.7)$ copies/ $\mu \mathrm{l}$ in non-HD stage $\mathrm{V}, p=$ 0.002) (Fig. 2a). Moreover, the levels of plasmatic $16 \mathrm{~S}$ rDNA in HD patients were similar to those of controls.

Since urea has been described as a gut toxic in CKD, we analyzed its variations: urea displayed increasing levels in non-HD stage $\mathrm{V}$ patients compared to controls (27.6 (22.7-30.9) in non-HD stage $\mathrm{V}$ vs $5.4(4.8-6.4)$ $\mathrm{mmol} / \mathrm{l}$ in controls, $p<0.0001)$ and similar levels in HD compared to non-HD stage V (19.3 (14.0-24.0) in HD patients vs $27.6(22.7-30.9) \mathrm{mmol} / \mathrm{l}$ in non-HD stage $\mathrm{V}$, $p=0.7$ ) (Fig. 2b). 16S rDNA levels were not correlated with urea $(\mathrm{p}=0.7)$.

To evaluate the possible role of uremic toxins produced by the gut microbiota in bacterial translocation and in microinflammation in CKD, IS, PCS and IAA plasmatic levels were measured for each group. IS, PCS and IAA increased with the stage of CKD and in HD (Fig. 2c-e). IS, PCS and IAA levels were higher in HD than in controls (106.3 (73.3-130.4) in HD patients vs 


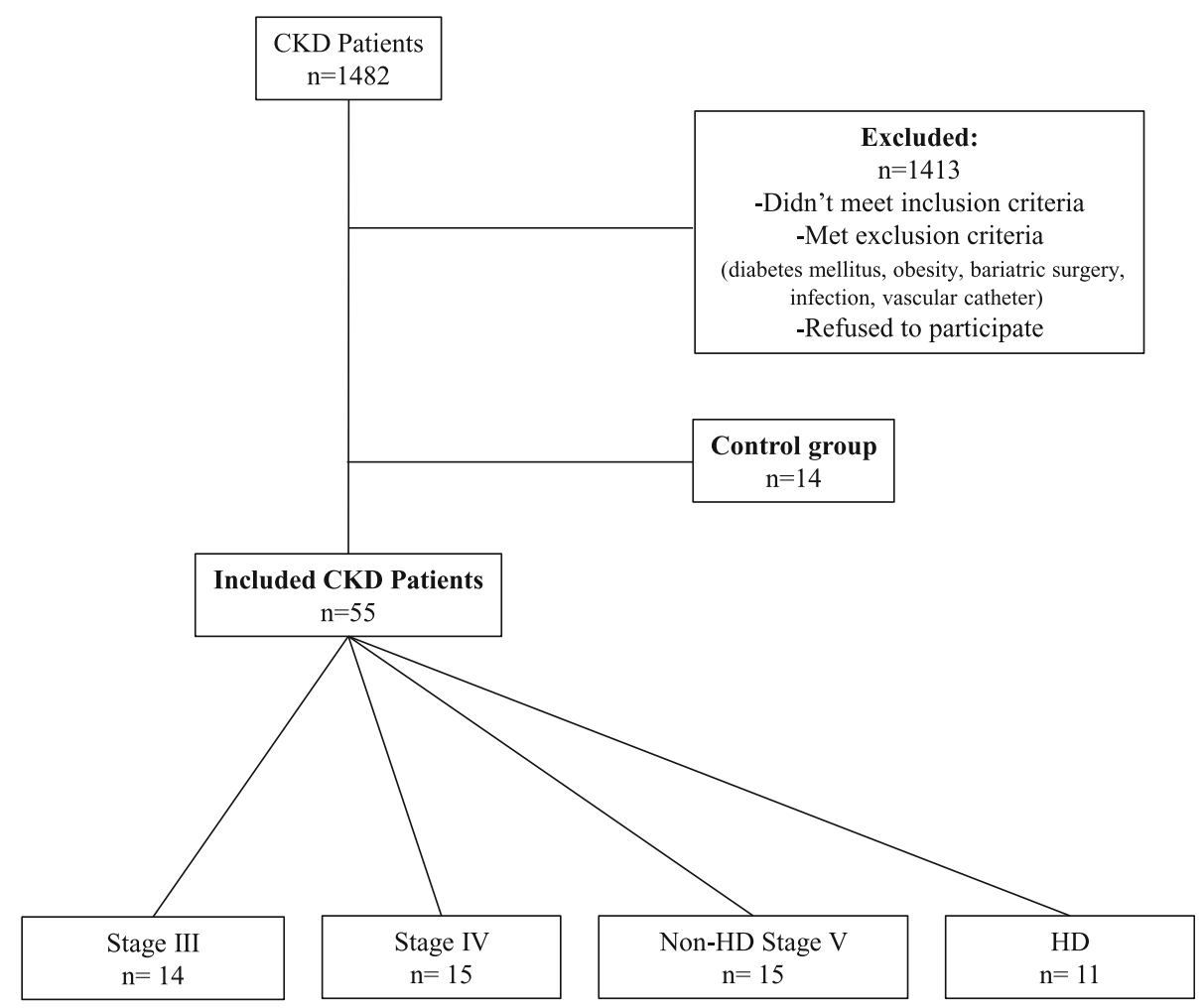

Fig. 1 Selection of the study population

$3.2(2.4-5.1) \mu \mathrm{mol} / \mathrm{l}$ in controls, $p<0.0001$ for IS; 174.2 (125.0-227.5) in HD patients vs $23.7(13.9-52.6) \mu \mathrm{mol} / \mathrm{l}$ in controls, $p=0.006$ for PCS; and $3.7(2.6-4.6)$ in HD patients vs $1.3(1.0-1.9) \mu \mathrm{mol} / \mathrm{l}$ in controls, $p=0.0002$ for IAA). To evaluate the role of oxidative stress in microinflammation, we measured plasmatic F2isoprostanes. However, F2-isoprostanes levels did not vary with the stage of CKD nor in HD patients (Fig. 2f).

\section{Microinflammation according to CKD stage}

To evaluate if microinflammation was related to bacterial translocation, uremic toxins, or to oxidative stress, we measured hsCRP and RITNF $\alpha$ in each group. RITNF $\alpha$ levels were higher in HD patients than in controls (12.6 (9.6-13.3) in HD patients vs $1.1(1.0-1.4) \mathrm{ng} / \mathrm{ml}$ in controls, $p<0.0001)$ and hsCRP levels were higher in nonHD stage $\mathrm{V}$ patients than in controls $(2.9$ (1.4-8.5) in non-HD stage $\mathrm{V}$ vs $0.8(0.5-1.7) \mathrm{mg} / \mathrm{l}$ in controls, $p=$ $0.01)$ and remained stable in HD patients (2.9 (1.4-8.5) in non-HD stage $\mathrm{V}$ vs $5.1(0.9-11.5) \mathrm{mg} / \mathrm{l}$ in HD patients, $p=1$ ) (Fig. 3a-b). Thus, microinflammation rather followed the course of uremic toxins IS, PCS and IAA, than that of bacterial translocation.

\section{Factors associated with microinflammation in CKD}

Then we determined the associations between microinflammation and bacterial translocation, uremic toxins and oxidative stress. $16 \mathrm{~s}$ rDNA or F2-isoprostanes were not correlated to microinflammation in CKD. IS was correlated with RITNF $\alpha,(r=0.8, p<0.0001)$, PCS and IAA were also correlated with RITNF $\alpha$ but to a lesser extent ( 0.6 and 0.5 respectively, $\mathrm{p}<0.0001$ ) (Fig. 4a, c, e). IS and PCS were significantly higher in patients with hsCRP $\geq 5 \mathrm{mg} / \mathrm{l}$ than in patients with hsCRP $<5 \mathrm{mg} / \mathrm{l}$ ( $p=$ 0.01 and $p=0.04$, respectively) (Fig. $4 \mathrm{~b}, \mathrm{~d}$ ). IAA levels were not significantly increased in patients with hsCRP $\geq 5 \mathrm{mg} / \mathrm{l}$ (Fig. 4f).

As IS, PCS and IAA are synthetized by proteolytic bacteria selected from the gut microbiota under high levels of intraluminal urea, we analyzed the association between plasmatic urea and microinflammation. Urea was correlated with RITNF $\alpha(\mathrm{r}=0.8, p<0.0001)$ and was significantly higher in patients with hsCRP $\geq 5 \mathrm{mg} / \mathrm{l}$ vs hsCRP $<5 \mathrm{mg} / \mathrm{l} \quad(p=0.01)$ (Fig. $4 \mathrm{~g}, \mathrm{~h})$, suggesting that urea could play a role in microinflammation in CKD.

\section{Discussion}

This study highlights that CKD patients present increased microinflammation markers even in the absence of diabetes mellitus, obesity, active infections, neoplastic or auto immune diseases. This microinflammation increases with the stage of CKD and even more in HD patients as has been already shown in literature [21]. We found that only uremic toxins IS, PCS and urea were 


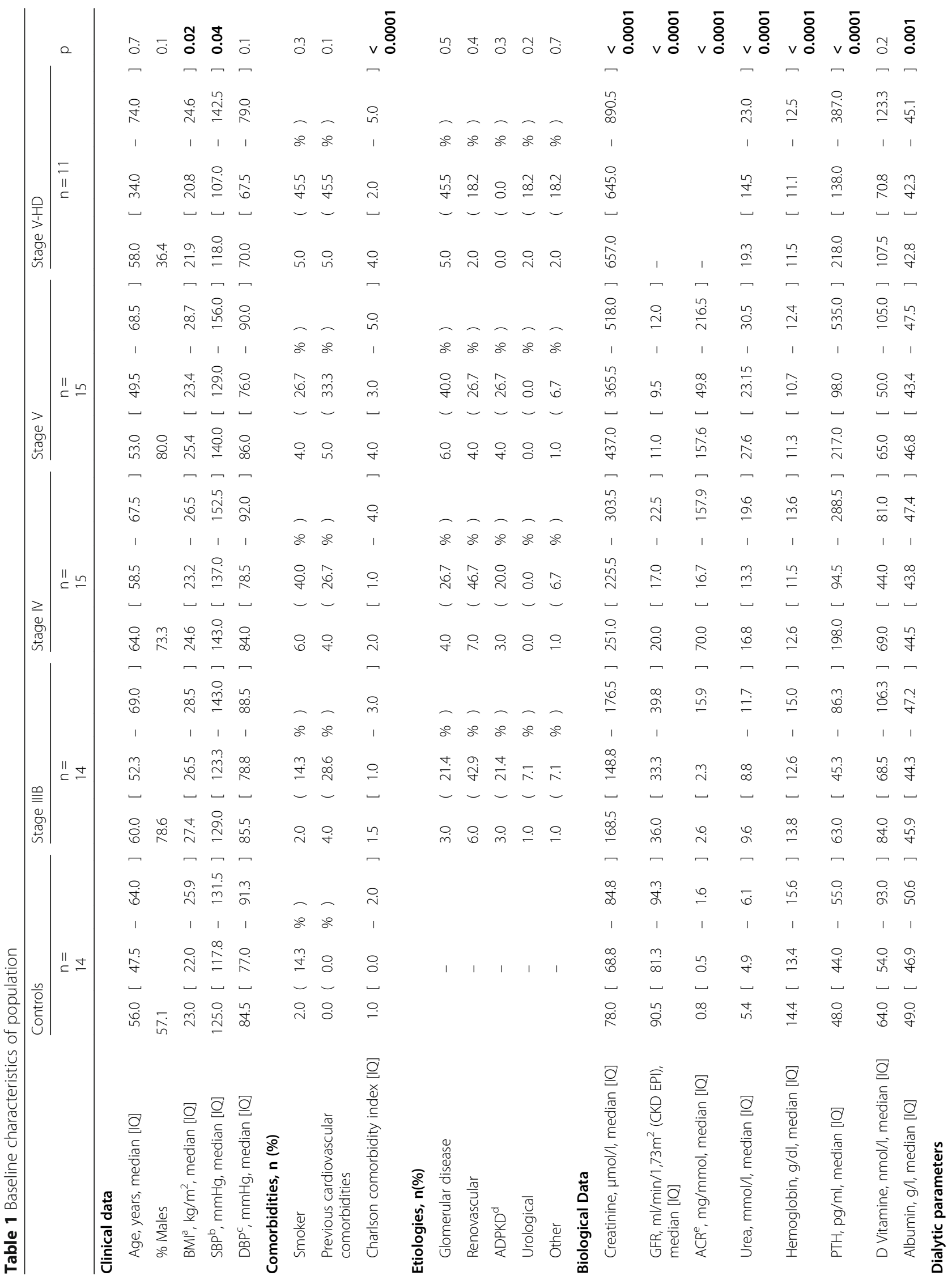




$$
\text { D }
$$


A

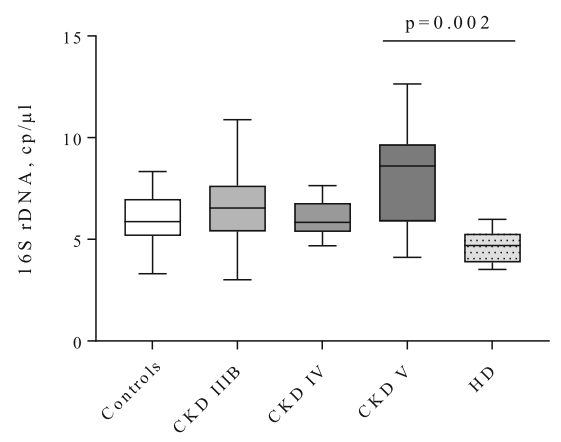

$\mathrm{C}$

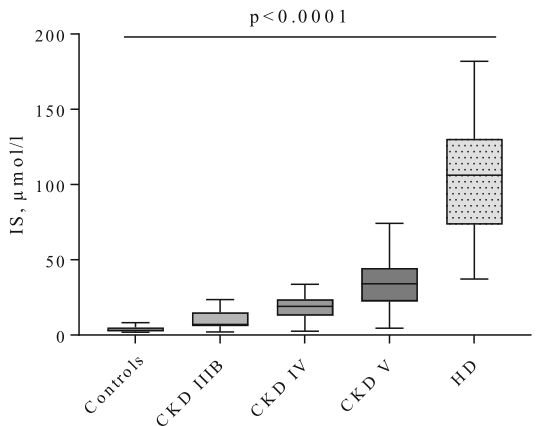

$\mathrm{E}$

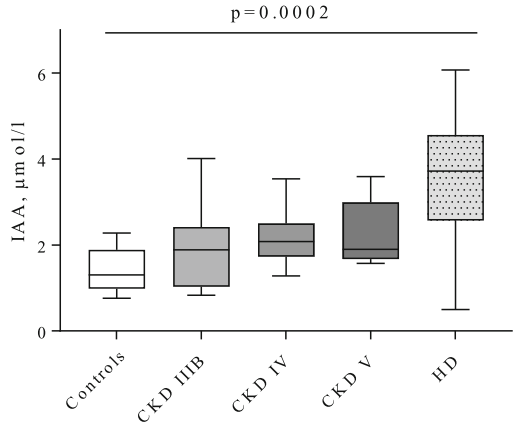

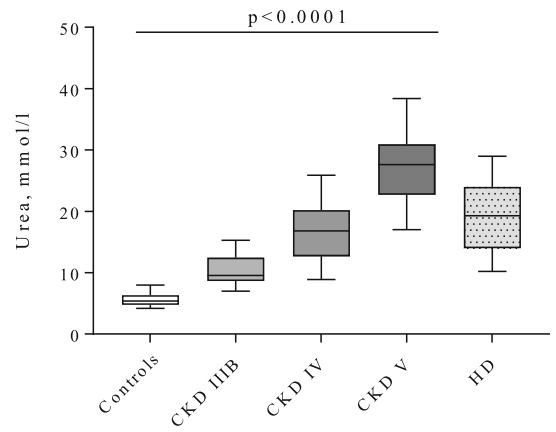

$\mathrm{D}$

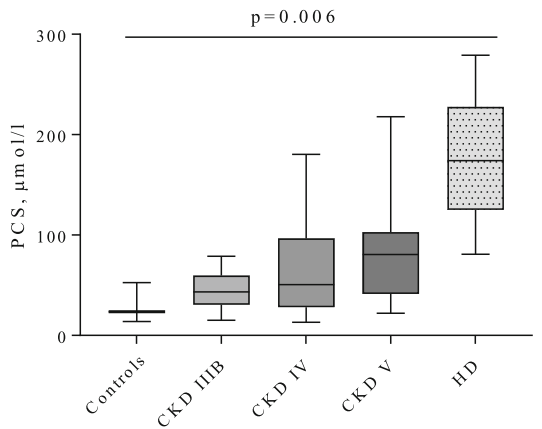

F

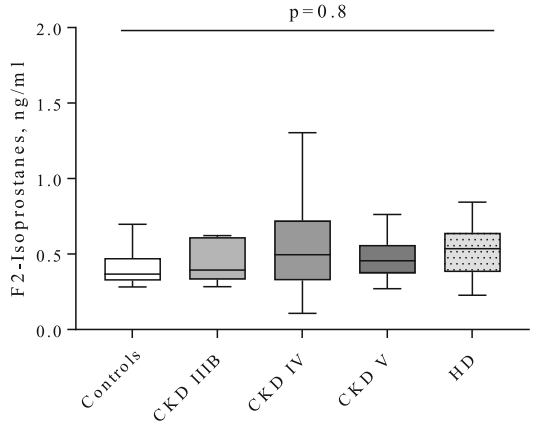

Fig 2 Bacterial translocation, urea, uremic toxins and oxidative stress according to CKD stage. a- Plasma 16S rDNA, copies/ $\mu$, b- plasma urea, mmol/I, c-plasma indoxyl sulfate (IS), $\mu \mathrm{mol} / \mathrm{l}, \mathbf{d}$ - plasma p-cresyl sulfate (PCS), $\mu \mathrm{mol} / \mathrm{l}$, e- plasma indole acetic acid (IAA), $\mu \mathrm{mol} / \mathrm{l}, \mathbf{f}-$ plasma F2isoprostanes, $\mathrm{ng} / \mathrm{I}$. Kruskal-Wallis statistic test with multipaire wise comparison from Dunn: $n=14$ for controls, $n=14$ for stage IIIB, $n=15$ for stage $I V, n=15$ for non-HD stage $\mathrm{V}, n=11$ for HD except for PCS: $n=3$ for controls, $n=12$ for stage IIIB, $\mathrm{n}=14$ for stage IV, $n=14$ for non-HD stage $\mathrm{V}$, $n=9$ for $\mathrm{HD}$

associated with microinflammation in CKD. Surprisingly, our CKD patients did not show any significant variation in the oxidative stress marker F2isoprostanes compared to controls. Bacterial translocation marker was not increased in non-HD patients compared to controls, and clearly decreased in HD patients compared to non-HD stage V.
To our knowledge, our study is the first translational study showing a decrease of bacterial translocation in HD patients compared to non-HD advanced stages of CKD. HD patients could be at risk of increasing bacterial translocation due to per dialytic episodes of hypotension, ultrafiltration, mesenteric ischemia, impaired cardiac function, reduction of splanchnic blood flow that impair 
A

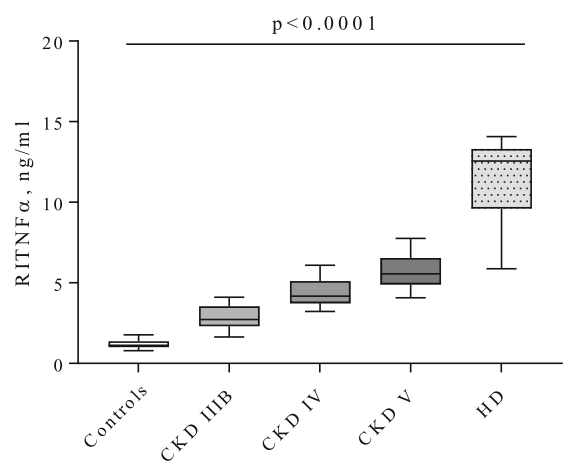

B

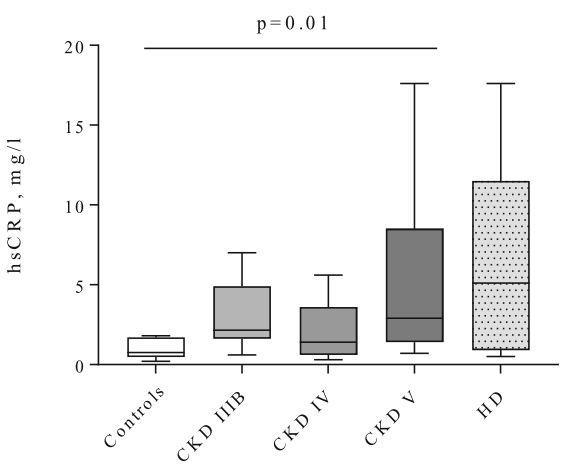

Fig. 3 Microinflammation according to CKD stage. a- RITNFa, ng/l, b- hsCRP, mg/l. Kruskal-Wallis statistic test with multipaire wise comparison from Dunn: $n=14$ for controls, $n=14$ for stage IIIB, $n=15$ for stage IV, $n=15$ for non-HD stage $V, n=11$ for HD

intestinal barrier [22]. Indeed, the study from McIntyre showed increased plasmatic rates of lipopolysaccharide in CKD patients and even more in patients under HD [9]. One reason that we found conflicting results is that we used plasmatic levels of bacterial $16 \mathrm{~S}$ rDNA which allowed us to detect all translocated bacteria from intestine to blood specifically if measured after a fasting period. Another reason is that we excluded patients with catheter and hemodynamic instability as only one HD patient displayed a perdialytic hypotension during the analyzed session. According to these results, microinflammation in our HD patients is not related to bacterial translocation.

The level of bacterial translocation was not increased in our non-HD CKD patients compared to controls. The patients did not display any known confounding factors of bacterial translocation such as diabetes mellitus [16], obesity [17], cardiac failure, HIV infection [23], inflammatory bowel disease [24], alcohol disorder [18] which could favor gut barrier permeability and thus lead to high plasmatic $16 \mathrm{~S}$ rDNA levels. Another point is that our CKD patients had a high vitamin D level, at any stage of the disease, probably due to an efficient supplementation. It has been shown that apart from its immune modulating effects, vitamin D influences digestive barrier quality. In vivo, it could restore gut permeability in a mouse model of colitis, acting on the tight junction proteins [25]. Furthermore, HIV and hepatitis $\mathrm{C}$ coinfected patients had a lower bacterial translocation if they had a 25-hydroxy vitamin $\mathrm{D}$ in the normal range compared to vitamin D deficient patients [26]. This could explain the fact that our patients had a low level of bacterial translocation. Another provocative hypothesis is that indoles could have a protective effect on the gut barrier. In a mouse model of colitis, low stool levels of IAA were associated with intestinal inflammation
[27]. High plasmatic levels of tryptophan metabolites like IAA in stage $\mathrm{V}$ non-HD and HD patients could activate aryl hydrocarbon receptors which regulate local IL-22 production for intestinal homeostasis [28] resulting in gut barrier protection. Interestingly, a recent study evaluated blood microbiome in non-diabetic CKD patients using 16S PCR: quantitative circulating $16 \mathrm{~S}$ rDNA did not differ between CKD patients and controls [29] supporting the fact that bacterial translocation is not increased in non-diabetic CKD patients. Of course, we also can not eliminate a lack of effect and a falsely unchanged rate of bacterial translocation, due to the low number of patients in this pilot study.

One key factor known to lead to the impairment of the gut barrier and to bacterial translocation in CKD is urea. Urea has been more described as a reflection of other toxic metabolites accumulation in CKD than a real uremic toxin. Albeit recently, urea has gained recognition as a toxic metabolite implied in endothelial and adipocyte dysfunction and in gut barrier impairment [30] as intraluminal urea increases in the intestine with decline of renal function. Urea is directly toxic on gut barrier [31], leading to endocytosis of tight junction proteins $[31,32]$ and to an increased gut permeability, and thus to an abnormal passage of bacteria or bacterial fragments from the gut lumen to the circulation [33], stimulating the immune system. In our study, plasmatic urea was not correlated to $16 \mathrm{~S}$ rDNA suggesting non-linear and more complex relationship between plasmatic and intraluminal urea and bacterial translocation. Surprisingly, bacterial translocation was not correlated with microinflammation in our study whereas urea was, meaning that urea could participate in microinflammation in CKD by another mechanism than altering gut permeability and increasing bacterial translocation. Thus, one hypothesis is that urea could stimulate 
Olivier et al. BMC Nephrology

(2020) 21:141

Page 9 of 11

A

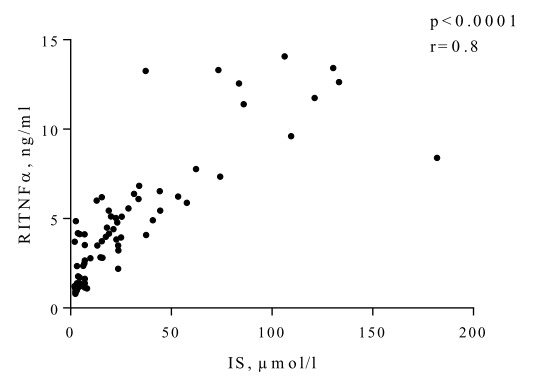

C

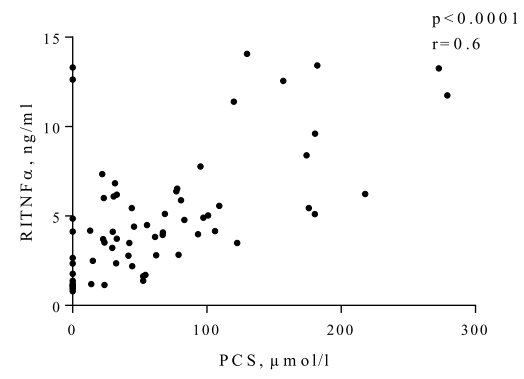

E

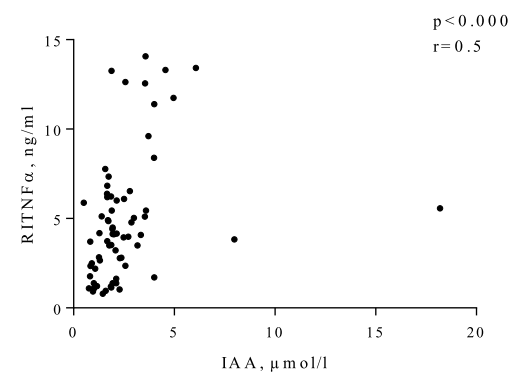

G

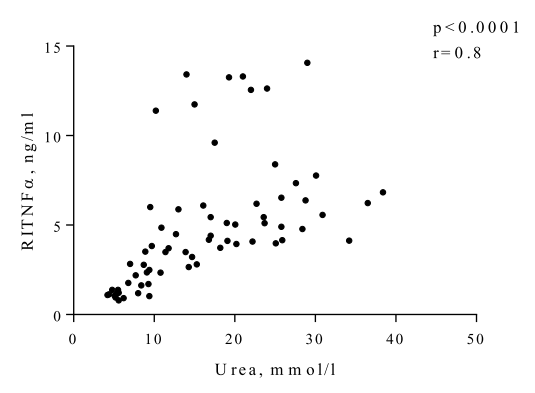

B

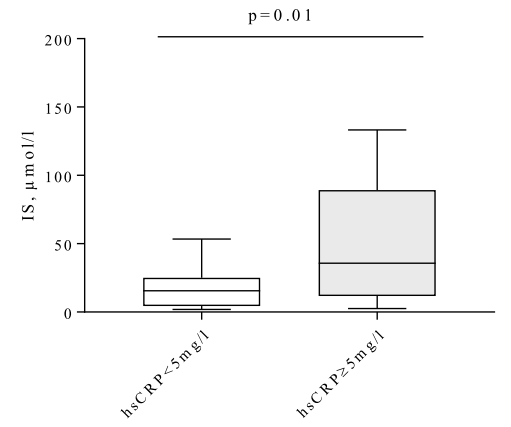

D

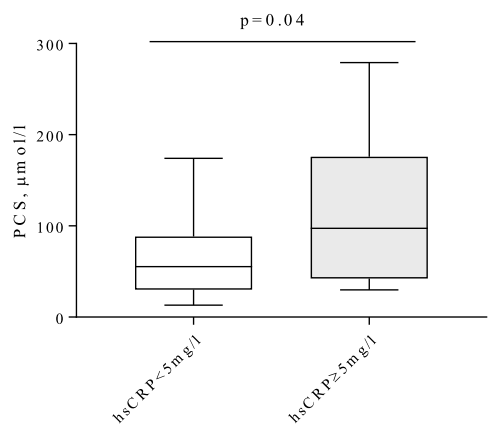

F

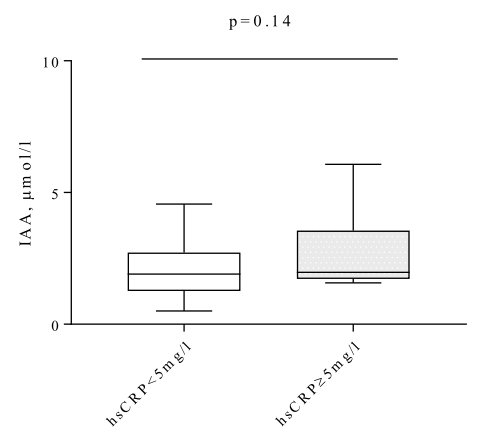

$\mathrm{H}$

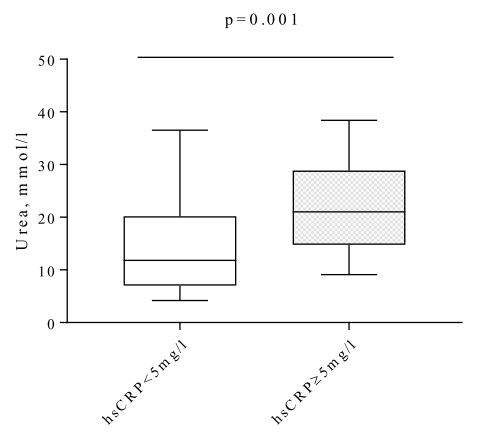

Fig 4 (See legend on next page.) 
(See figure on previous page.)

Fig 4 Factors associated with microinflammation in CKD. a- Correlation between indoxyl sulfate (IS) and RITNFa, c-correlation between p-cresyl sulfate (PCS) and RITNFa, e- correlation between indole acetic acid (IAA) and RITNFa, $\mathbf{g}$-correlation between urea and RITNFa. $\mathbf{b}$ - IS levels in subgroups with hsCRP < or $\geq 5 \mathrm{mg} /$. d - PCS levels in subgroups with hsCRP $<$ or $\geq 5 \mathrm{mg} /$. f- $\mathrm{f}$ IAA levels in subgroups with hsCRP $<$ or $\geq 5 \mathrm{mg} /$. h- Urea levels in subgroups with hsCRP $<$ or $\geq 5 \mathrm{mg} / \mathrm{l}$. Spearman correlation test for $A, C, E, G$ and Mann-Whitney test for $B, D, F, H: n=51$ for hsCRP $<5 \mathrm{mg} / \mathrm{l}, n=18$ for hsCRP $\geq 5 \mathrm{mg} / \mathrm{l}$, except for PCS: $n=37$ for hsCRP $<5 \mathrm{mg} / \mathrm{l}, \mathrm{n}=15$ for hsCRP $\geq 5 \mathrm{mg} / \mathrm{l}$

microinflammation by the selection of IS, PCS, IAA producing bacteria in gut microbiota but this hypothesis cannot be confirmed in this pilot study.

Our CKD patients did not show any significant increase in plasmatic F2-isoprostanes compared to controls. It is important to note that oxidative stress is difficult to assess in vivo. There is no reference marker to quantify oxidative stress in routine, and literature presents many different markers without any consensus [34]. Among all those markers, quantification of plasmatic F2-isoprostanes by mass spectrometry appeared to be the most reliable to measure oxidative stress associated with CKD in patients [35]. Interestingly, our study is the first, to our knowledge, to describe oxidative stress in a population of CKD patients, without metabolic diseases and without hemodynamic instability in HD. As a result, we cannot conclude that oxidative stress is a major determinant in microinflammation in CKD patients without metabolic comorbidities.

Our study showed a positive association between microinflammation markers and uremic toxins such as IS at any CKD stage. This toxin is known to take a part in immune and endothelial cell activation in CKD. IS was described as being a direct actor in atherosclerosis development, enhancing the production of microparticles by activated endothelial cells [36], and inhibiting in vitro the proliferation and reparation of endothelium $[37,38]$. Furthermore IS could simulate the synthesis of proinflammatory cytokines by monocytes such as IL-6 and IL-1 [39]. Increased IS has been described as a risk factor of vascular calcification, arterial stiffness and cardiovascular mortality in a population of $\mathrm{HD}$ and non-HD CKD patients. In our work, the toxins IS, PCS and IAA were increased in CKD patients and even more in HD patients, suggesting that they are poorly eliminated in HD. These toxins are protein bound and can only be eliminated by adsorption in HD therefore other therapeutics aiming at decreasing these toxins can be meaningful. The spherical carbon adsorbent of indole AST-120 has been shown to reduce IS serum levels and improve uremic symptoms such as malaise [40] in CKD patients. Unfortunately AST-120 has shown no beneficial effect on the progression of CKD in the EPPIC trials [41].

The main limitations of our monocentric study are the cross sectional study design, the low number of subjects, limiting analyses with lack of power. The variability of samples did not allow us to obtain clear subgroup analysis especially for bacterial translocation, therefore we cannot eliminate a link between bacterial translocation and microinflammation in non-HD stage V. A longitudinal and more powerful study could be interesting to precise bacterial translocation evolution in CKD. However, our data allowed us to raise hypothesis about microinflammation caused only by reduced renal function in a strictly selected CKD population.

\section{Conclusions}

We showed that CKD patients without any associated metabolic or inflammatory diseases presented high markers of inflammation, particularly in HD. Bacterial translocation was unchanged in CKD patients compared to controls but clearly decreased under HD treatment compared to late stage of CKD. Microbiota derived uremic toxins such as IS could definitely play an important role in microinflammation and atherosclerosis development in those patients.

\section{Abbreviations}

BMI: Body mass index; CKD: Chronic kidney disease; HD: Hemodialysis; IAA: Indole acetic acid; IS: Indoxyl sulfate; NADPH: Nicotinamide adenin dinucleotide phosphatase; PCS: P-cresyl sulfate; RITNFa: Receptor I of TNFa; SBP: Systolic blood pressure

\section{Acknowledgements}

We thank CDR, PC, and JPL, who belong to the FHU INCh (Federation Hospitalo Universitaire infections Chroniques, Aviesan). We thank the Nîmes University hospital for its structural and human support.

\section{Authors' contributions}

O.M., C.D.R. and V.O. designed the study, V.O., C.D.R., P.C., J.P.C., TS and S.B. carried out experiments, C.D.R, O.M. and V.O. analyzed the data, V.O. made the figures and drafter the paper, O.M., C.D.R., S.B., J.P.C., P.C. and J.P.L. revised the paper, all authors approved the final version of the manuscript.

\section{Funding}

This study has been supported by University hospital of Nimes through the award obtained during the internal call for tenders "Thématiques phares et Thématiques émergentes" and INSERM. These organizations had no role in study design, collection, analysis, interpretation of data, or in writing the article.

\section{Availability of data and materials \\ The datasets used during the current study are available from the corresponding author on reasonable request.}

\section{Ethics approval and consent to participate}

This study was approved by the institutional review board (attributed number: 16.11.03) and carried out in accordance with the Helsinki

Declaration as revised in 2008. All patients gave oral informed consent to participate in the study, as required by the administration according to the opinion of the Ethics committee. 


\section{Consent for publication}

Not applicable.

\section{Competing interests}

Olivier Moranne is associated editor of the BMC Nephrology Journal.

\section{Author details}

'Department of Nephrology - Dialysis - Apheresis, Caremeau Hospital, University Montpellier-Nîmes, CHU Nîmes, Nimes, France. ${ }^{2}$ Department of Microbiology and Hospital Hygiene, U1047, INSERM, University of Montpellier, CHU Nîmes, Nîmes, France. ${ }^{3}$ UMR9002, Institute for Human Genetics, CNRS-University of Montpellier, Montpellier, France. ${ }^{4}$ PhyMedExp, INSERM, CNRS, University of Montpellier, Montpellier, France. ${ }^{5}$ Department of Biochemistry and Hormonology, CHU Montpellier, Montpellier, France. ${ }^{6}$ C2VN, INSERM 1263, INRA 1260, Aix-Marseille University, Marseille, France. ${ }^{7}$ EA2415, Laboratoire Epidémiologie, Santé Publique, Biostatistiques, University of Montpellier, Nîmes, France.

\section{Received: 30 December 2019 Accepted: 12 April 2020}

Published online: 21 April 2020

\section{References}

1. Astor BC, Hallan SI, Miller ER, Yeung E, Coresh J. Glomerular filtration rate, albuminuria, and risk of cardiovascular and all-cause mortality in the US population. Am J Epidemiol. 2008;167:1226-34.

2. Ridker PM, et al. Antiinflammatory therapy with Canakinumab for atherosclerotic disease. N Engl J Med. 2017;377:1119-31.

3. Thompson $\mathrm{S}$, et al. Cause of death in patients with reduced kidney function. J Am Soc Nephrol. 2015;26:2504-11.

4. Van Linthout S, Tschöpe C. Inflammation - cause or consequence of heart failure or both? Curr Heart Fail Rep. 2017;14:251-65.

5. Kimmel PL, et al. Immunologic function and survival in hemodialysis patients. Kidney Int. 1998:54:236-44.

6. Sterling KA, Eftekhari P, Girndt M, Kimmel PL, Raj DS. The immunoregulatory function of vitamin D: implications in chronic kidney disease. Nat Rev Nephrol. 2012:8:403-12.

7. Kraut JA, Madias NE. Consequences and therapy of the metabolic acidosis of chronic kidney disease. Pediatr. Nephrol Berl Ger. 2011;26:19-28.

8. Shoelson SE, Lee J, Goldfine AB. Inflammation and insulin resistance. J Clin Invest. 2006;116:1793-801.

9. Mclntyre CW, et al. Circulating endotoxemia: a novel factor in systemic inflammation and cardiovascular disease in chronic kidney disease. Clin J Am Soc Nephrol. 2011;6:133-41.

10. Lau WL, Kalantar-Zadeh K, Vaziri ND. The gut as a source of inflammation in chronic kidney disease. Nephron. 2015;130:92-8.

11. Jourde-Chiche $N$, et al. Protein-bound toxins--update 2009. Semin Dial. 2009;22:334-9.

12. Morena $\mathrm{M}$, et al. Oxidative stress in hemodialysis patients: is NADPH oxidase complex the culprit? Kidney Int Suppl. 2002:109-14. https://doi.org/10.1046/ j.1523-1755.61.s80.20.x.

13. Ruiz S, Pergola PE, Zager RA, Vaziri ND. Targeting the transcription factor Nrf2 to ameliorate oxidative stress and inflammation in chronic kidney disease. Kidney Int. 2013;83:1029-41.

14. Kuchta A, et al. Estimation of oxidative stress markers in chronic kidney disease. Kidney Blood Press Res. 2011;34:12-9.

15. Mao Y-H, et al. The ratio of plasma and urinary 8-oxo-Gsn could be a novel evaluation index for patients with chronic kidney disease. Oxidative Med Cell Longev. 2018;2018:4237812.

16. Cani PD, et al. Metabolic endotoxemia initiates obesity and insulin resistance. Diabetes. 2007:56:1761-72.

17. Ortiz $\mathrm{S}$, et al. Bacterial DNA translocation holds increased insulin resistance and systemic inflammatory levels in morbid obese patients. J Clin Endocrinol Metab. 2014;99:2575-83.

18. Donnadieu-Rigole $\mathrm{H}$, et al. Beneficial effect of alcohol withdrawal on gut permeability and microbial translocation in patients with alcohol use disorder. Alcohol Clin Exp Res. 2017. https://doi.org/10.1111/acer.13527.

19. Calaf $R$, et al. Determination of uremic solutes in biological fluids of chronic kidney disease patients by HPLC assay. J Chromatogr B Analyt Technol Biomed Life Sci. 2011;879:2281-6.

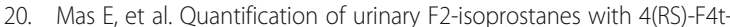
neuroprostane as an internal standard using gas chromatography-mass spectrometry application to polytraumatized patients. J Chromatogr B Analyt Technol Biomed Life Sci. 2008:872:133-40.

21. Combe C. Dialysis dose, nutrition, inflammation: what is the relationship? Data from the Dialysis outcomes and practices patterns study (DOPPS). Néphrologie Thérapeutique. 2010;6:7-12.

22. Jakob SM, Ruokonen E, Vuolteenaho O, Lampainen E, Takala J. Splanchnic perfusion during hemodialysis: evidence for marginal tissue perfusion. Crit Care Med. 2001;29:1393-8.

23. Abad-Fernández $\mathrm{M}$, et al. Correlation between different methods to measure microbial translocation and its association with immune activation in long-term suppressed HIV-1-infected individuals. J Acquir Immune Defic Syndr. 2013;1999(64):149-53.

24. Vrakas $\mathrm{S}$, et al. Intestinal Bacteria composition and translocation of Bacteria in inflammatory bowel disease. PLoS One. 2017;12:e0170034.

25. Assa $A$, et al. Vitamin D deficiency predisposes to adherent-invasive Escherichia coli-induced barrier dysfunction and experimental colonic injury. Inflamm Bowel Dis. 2015;21:297-306.

26. García-Álvarez M, et al. Optimal vitamin D plasma levels are associated with lower bacterial DNA translocation in HIV/hepatitis c virus coinfected patients. AIDS Lond Engl. 2016;30:1069-74.

27. Lamas B, et al. CARD9 impacts colitis by altering gut microbiota metabolism of tryptophan into aryl hydrocarbon receptor ligands. Nat Med. 2016;22 598-605.

28. Zelante T, et al. Tryptophan catabolites from microbiota engage ary hydrocarbon receptor and balance mucosal reactivity via interleukin-22. Immunity. 2013:39:372-85.

29. Shah NB, et al. Blood microbiome profile in CKD : a pilot study. Clin J Am Soc Nephrol. 2019;14:692-701.

30. Lau WL, Vaziri ND. Urea, a true uremic toxin: the empire strikes back. Clin Sci Lond Engl. 2017;1979(131):3-12.

31. Vaziri ND, Yuan J, Norris K. Role of urea in intestinal barrier dysfunction and disruption of epithelial tight junction in chronic kidney disease. Am J Nephrol. 2013:37:1-6.

32. Vaziri ND, et al. Uremic plasma impairs barrier function and depletes the tight junction protein constituents of intestinal epithelium. Am J Nephrol. 2012;36:438-43

33. Vaziri ND, et al. Disintegration of colonic epithelial tight junction in uremia: a likely cause of CKD-associated inflammation. Nephrol Dial Transplant. 2012;27:2686-93.

34. Frijhoff J, et al. Clinical relevance of biomarkers of oxidative stress. Antioxid Redox Signal. 2015;23:1144-70.

35. Briskey DR, Wilson GR, Fassett RG, Coombes JS. Optimized method for quantification of total $F$ (2)-isoprostanes using gas chromatography-tandem mass spectrometry. J Pharm Biomed Anal. 2014;90:161-6.

36. Faure $V$, et al. Elevation of circulating endothelial microparticles in patients with chronic renal failure. J Thromb Haemost. 2006:4:566-73.

37. Dou L, et al. The uremic solutes $p$-cresol and indoxyl sulfate inhibit endothelial proliferation and wound repair. Kidney Int. 2004:65:442-51.

38. Dou L, Burtey S. The harmful effect of indoxyl sulfate on neovascularization in chronic kidney disease. Kidney Int. 2016;89:532-4.

39. Sallée $M$, et al. The aryl hydrocarbon receptor-activating effect of uremic toxins from tryptophan metabolism: a new concept to understand cardiovascular complications of chronic kidney disease. Toxins. 2014:6:934-49.

40. Schulman $\mathrm{G}$, et al. A multicenter, randomized, double-blind, placebo-controlled, dose-ranging study of AST-120 (Kremezin) in patients with moderate to severe CKD. Am J Kidney Dis Off J Natl Kidney Found. 2006;47:565-77.

41. Schulman G, et al. Randomized placebo-controlled EPPIC trials of AST-120 in CKD. J Am Soc Nephrol. 2015;26:1732-46.

\section{Publisher's Note}

Springer Nature remains neutral with regard to jurisdictional claims in published maps and institutional affiliations. 